\title{
A New Procedure for analysis and Modelling of Male Urine Flow Rate
}

\author{
RUI Li ${ }^{1}$, ANDREW Gammie ${ }^{2}$, ZHU Quanmin ${ }^{1}$, MOKHTAR Nibouche ${ }^{1}$, JANICE Kiely $^{1}$ \\ 1. Faculty of Environment and Technology, University of the West of England, Frenchay Campus, Coldharbour Lane, Bristol, BS16 \\ 1QY, UK \\ E-mail: rui2.li@live.uwe.ac.ukquan.zhu@uwe.ac.uk Mokhtar.Nibouche@uwe.ac.uk Janice.Kiely@uwe.ac.uk
}

\section{The Bristol Urological Institute, Southmead Hospital, Bristol, BS10 5NB, UK}

E-mail: Andrew.Gammie@bui.ac.uk

\begin{abstract}
In the first stage studies, this paper proposes a new piecewise linear discrete time dynamic model for describing global urine flow rate profiles against time sequence. The establishment of model has been guided by physician meaningful principles and measured data. To obtain the model parameters from measured data sequence, a number of existing approaches have been tailored/integrated including least squares algorithm for model time constant and gain estimation, Butterworth filter with specified cut off frequencies, for reducing noise effect induced by abdominal and bladder squeezing, then to facilitate counting the number of peaks. A few of real case studies are selected to demonstrate the effectiveness and efficiency of the proposed procedure.
\end{abstract}

Key Words: Urine flow rate model, urodynamic, Butterworth filter, shape urine flow rate

\section{Introduction}

With worldwide population growing, the ratio of aging population has raised continuously. There is an increased number of patients linked to urinary system diseases, especially for male. Urodynamic tests have been predominantly used to check the function of the bladder and help to investigate the cause of urinary incontinence. Urine flow rate is one of the most significant parameters for assisting physicians to diagnose urinary system status/symptoms, such as normal, underactive and obstructive. In the meanwhile, various current urodynamic tests still remain an invasive way for recording the pressure and flow rate by inserting tubes through urinary tract to patient's bladder, which is painful and stressful for patients and causes test result inaccurate. Furthermore, there are a large amount of artefacts while a patient takes the urodynamic test, causing massive fluctuations in the urine flow rate curve, so that these add uncertainty in diagnose when a physician tries to analyse the urine flow rate curve. For instance, even an experienced physician may have a quarter misdiagnose in distinction between underactive and bladder outlet obstruction. To reduce such disturbances in measurement, non-invasive tests have been adopted [1-5].

In the last two decades, there have been several noninvasive measurement based urodynamic models have been set up [1-5]. Started from 1989 [7, 8] and formally established in 2013, a mathematical model called VBN (the initials of the first three authors) [6] has been applied to various studies, which is useful for the modelling of urodynamic function but has some intrinsic limitations, such as limited descriptions of sudden entry of the urine into the urethra and abdominal pressure. The other

This work is partially supported by the Bristol Urological Institute, UK, Department of Engineering Design and Mathematics, University of the West of England under PG travel grant, the National Nature Science Foundation of China under Grant 61273188 and Taishan Scholar Funding, Shandong, China. representative urodynamic model by analysing of detrusor pressure-flow measurements with the aid of the IBM compatible software package CLIM [9] was proposed in 1991, however it has been observed to have quarter inaccuracy from using the existing software package to analyse those measured data in obstructed cases. In general most of the current non-invasive methods have used complicated mathematical models of bladder but had limitation in utilization. Therefore, development of a concise and physician meaningful model has been a widely demanded research and clinical practice.

To justify the study on the above challenging issues, a few of research questions are listed below, which subsequently guides the study to provide possible solutions.

Research question one: Can a concise and physician meaningful global urine flow rate model be proposed with good fitting to measure data?

Research question two: Is there any method can reduce the artefacts caused by squeezing but remain the true characteristics of the urine flow rate curve?

Research question three: Is a model obtained from the data sequence with reducing artefacts to improve accuracy compared with the model obtained from raw data?

With such insight, this study proposes a new Urine flow rate Model, where the model has concise and physician meaningful structure and the parameters in the model can be estimated by the Least Squares algorithms with measured data. To reduce the noise effect with the measured data, a Butterworth filter is adopted and selection of its cut-off frequency is linked to the explanation of physician meanings. Consequently the artefact caused by abdominal squeezing and bladder squeezing can be effectively reduced. Compare to previous method, proposed urine flow rate model mainly focus on the modelling and noise reducing instead of the bladder model establishment.

The rest of the studies are organised with model structure determination and parameters estimation (Section 2 ), filter design and test (Section 3), and summary of the study and future research (Section 4). 


\section{Urine flow rate model and parameter estimation}

This section proposes a new global urine flow rate model to describe whole urine process which consists two piecewise linear dynamic sub-models. Consequently it tailors a least squares algorithm to be able to estimate the model parameters from measured data.

\subsection{Urine flow rate model}

To understand the urodynamic for modelling, inspect a measured typical normal male urine flow rate curve in Figure 1. It can be observed the shape likes a bell added with slight fluctuations. Deviation from the normal shape could suggest some problems in urine flow process.

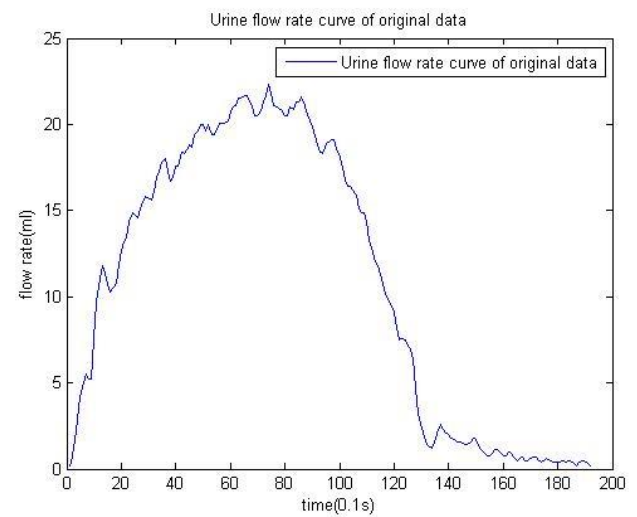

Figure 1 Measured normal urine flow rate curve

The observation gives guidance in determining a model structure with the following characteristics 1) it is a dynamic again time that could be represented by first order dynamic principle, 2) it is piecewise linear that has physician meaning to separate a urine process into upward rate flow session and downward rate flow session from the pick value (that is the top of the bell shape), which the time instance arriving at the pick value is defined as ridge point in the following descriptions.

Accordingly the global urine flow rate model is proposed as

$Q(t)=\left\{\begin{array}{lll}Q_{1}(t)=a_{1} Q_{1}(t-1)+b_{1} P_{1}(t) & t \leq n_{r} & \text { upward } \\ Q_{2}(t)=a_{2} Q_{2}(t-1)+b_{2} P_{2}(t) & t \succ n_{r} & \text { downward }\end{array}\right.$

where $t(1,2, \ldots)$ is the discrete time index, model output $Q_{i}(i=1,2)$ and model input $P_{i}(i=1,2)$ are the observed urine flow rate value and the virtual input value respectively, parameter $a_{i}(i=1,2)$ are linked to the corresponding time constants of the associated dynamic models and parameters $b_{i}(i=1,2)$ are the gain associated with the inputs, the coordinate $\left(n_{r}, Q_{r}\right)$ is the ridge point time index and the value. It should be explained that the model parameters have physician meanings and can be estimated from measured data. In dynamic principle time constant value determines how quickly a urine process moves toward to steady state, the greater of time constant value, the lower speed urine flow rate can be observed. It has been observed that even two patients have different urine flow rate curves and the maximum values, the proximate time constant value still can indicates these two patients may have the same symptom. Also the time constant value can be related with the pressure of the detrusor, and links with the pressure in the bladder.

\subsection{Model parameter estimation}

The work of model parameter estimation is to obtain the parameters $a_{i}(i=1,2)$ and $b_{i}(i=1,2)$ in model of $(2.1)$ by using a statistic algorithm with measured data. For this research, a classical Least Squares (LS) algorithm [10] is tailored to implement the data driven computations.

Consider a general linear in parameters regression model $y(t)=\varphi^{T}(t) \theta+e(t) \quad t=1,2, \ldots, N$

where $t$ is a discrete time index, $N$ is the length of measured data sequence, dependent variable $y(t)$ is a measurable quantity, regression

variable $\varphi^{T}(t)=\left[\begin{array}{llll}\varphi_{1}(t) & \varphi_{2}(t) & \cdots & \varphi_{L}(t)\end{array}\right]$ is a measurable L-vector, and error variable $e(t)$ is an unmeasurable quantity to represent modelling error caused from various factors such as measurement noise and external disturbance, and parameter vector $\theta=\left[\begin{array}{llll}\theta_{1} & \theta_{2} & \cdots & \theta_{L}\end{array}\right]^{T}$ is a L-vector to be estimated from measured $y(t)$ and $\varphi(t)$ in terms of the least squares errors, the difference between measured dependent variable $y(t)$ and model output variable $\varphi^{T}(t) \theta$.

In the computation algorithm, to find an estimate $\hat{\theta}$ of the parameter vector $\theta$ from measurements $y(1) \quad \varphi(1) \quad \cdots \quad y(N) \quad \varphi(N)$, a set of linear equations are formed, namely,

$$
\begin{gathered}
y(1)=\varphi^{T}(1) \theta \\
y(2)=\varphi^{T}(2) \theta \\
\vdots \\
y(N)=\varphi^{T}(N) \theta
\end{gathered}
$$

This can be written in matrix notation as

$$
Y(N)=\Phi(N) \hat{\theta}
$$

Where

$Y=\left[\begin{array}{c}y(1) \\ \vdots \\ y(N)\end{array}\right] \Phi=\left[\begin{array}{c}\varphi^{T}(1) \\ \vdots \\ \varphi^{T}(N)\end{array}\right]$

The normal equations take the form

$\left[\Phi^{T} \Phi\right] \theta=\Phi^{T} Y$

Therefore the estimation for the parameters can be determined by

$\hat{\theta}=\left[\Phi^{T} \Phi\right]^{-1} \Phi^{T} Y$ 
In regarding to the research, the parameter estimation work includes 1) by inspecting measured urine flow rate data sequence, identify the ridge point coordinate $\left(n_{r}, Q_{r}\right)$, which split the sequence into upward and downward subsequences, 2) with reference to $\left(n_{r}, Q_{r}\right)$, setup virtual stimulate inputs for each sub-sequence, 3) form the associated matrices and vectors from each of the subsequences, 4) use equation (2.6) to calculate the parameter vectors. The step by step procedure is illustrated below.

1) Let $\boldsymbol{N}$ be the measured data sequence, determine ridge point coordinate $\left(n_{r}, Q_{r}\right)$

2) Setup virtual step stimulate inputs

For upwards session $\left(t=1 \cdots n_{r}\right)$, setup $P_{1}(t)$ as a step input $n_{r} * 1$ vector with amplitude of $Q_{r}$ and zero initial, which represents a urine flow process driven by an internal force from bladder. Accordingly the sub-data sequence is formed as

$P_{1}(t)^{T}=\left[\begin{array}{lll}1 & \cdots & 1\end{array}\right]$

For downward session $\left(t=n_{r} \cdots N\right)$, setup $P_{2}(t)$ as a step input $\left(N-n_{r}\right) * 1$ vector with amplitude of 0 and initial amplitude of $Q_{r}$, which represents the remaining session of a urine flow process after diminish of the internal force from bladder. Accordingly the sub-data sequence is formed as

$P_{2}(t)^{T}=\left[\begin{array}{lllll}0 & 0 & 0 & \cdots & 0\end{array}\right]$

3) Form normal matrices $\Phi_{i}(i=1,2)$ and output vectors $Y_{i}(i=1,2)$

$\Phi_{1}=\left[\begin{array}{cc}q(1) & p(2) \\ \vdots & \vdots \\ q\left(n_{r}-1\right) & p\left(n_{r}\right)\end{array}\right] \quad \Phi_{2}=\left[\begin{array}{cc}q\left(n_{r}-1\right) & p\left(n_{r}\right) \\ \vdots & \vdots \\ q(N-1) & p(N)\end{array}\right]$

and

$Y_{1}=\left[\begin{array}{c}q(1) \\ \vdots \\ q\left(n_{r}-1\right)\end{array}\right] \quad Y_{2}=\left[\begin{array}{c}q\left(n_{r}\right) \\ \vdots \\ q(N)\end{array}\right]$

Let the parameter vectors be expressed as

$$
\theta_{1}=\left[\begin{array}{l}
a_{1} \\
b_{1}
\end{array}\right] \quad \theta_{2}=\left[\begin{array}{l}
a_{2} \\
b_{2}
\end{array}\right]
$$

4) Then substitute the formed matrices and vectors into equation (2.7) to obtain the parameter estimates.

\subsection{Calculation of time constants}

Time constant is defined by a first order Laplace transfer function (a representative to a first order linear differential equation) below [11].

$$
\frac{Q_{i}(s)}{P_{i}(s)}=\frac{k_{i}}{T_{i} s+1} \quad i=1,2
$$

where $s$ is the Laplace operator, $Q_{i}(s)$ and $P_{i}(s)$ are the Laplace transforms of the observed urine flow rate value and the virtual input value in terms of continuous time, respectively. $T_{i}$ is defined as time constant.

The proposed model (2.1) is in form of discrete time description and it corresponding $\mathrm{Z}$ transform (a representative to a first order linear difference equation) can be expressed as

$$
\frac{Q_{i}(\mathrm{z})}{P_{i}(\mathrm{z})}=\frac{b_{i}}{1+a_{i} z^{-1}} \quad i=1,2
$$

With reference to residue theorem to convert to continuous transfer function $F(s)$ from discrete time transfer function $F(z)$

$$
\begin{aligned}
& F(s)=\sum \operatorname{Re} s\left[F(z) \frac{z^{-1}}{s-\frac{1}{T_{s}} \ln z}\right] \\
& =\sum\left\{\frac{1}{(m-1) !} \frac{d^{m-1}}{d z^{m-1}}\left[\left(z-z_{i}\right)^{m} F(z) \frac{z^{-1}}{s-\frac{1}{T_{s}} \ln z}\right]\right\}_{z=z_{i}}
\end{aligned}
$$

where normalised sampling period $T_{s}=1$. For the proposed discrete time first order urine flow rate model, $\sum=1, m=1$, $z_{i}=a_{i}(i=1,2)$ and then the time constants of the continuous time model (2.19) can be obtained by

$T_{i}=-\frac{1}{\ln a_{i}} \quad i=1,2$

Therefore from the above analysis, the procedure to obtain the time constants of model (2.1) is consist of two steps 1) estimate $a_{i}(i=1,2)$ of model (2.1) from measured data and 2) calculate $T_{i}$ from the relationship of (2.16).

Example 1, To demonstrate the modelling procedure, chose the data shown in Figure 1. Follow the above steps, the resultant plots are shown in Figure 2. The fluctuated line and smooth line stand for original urine flow rate curve and estimated model output plot respectively.

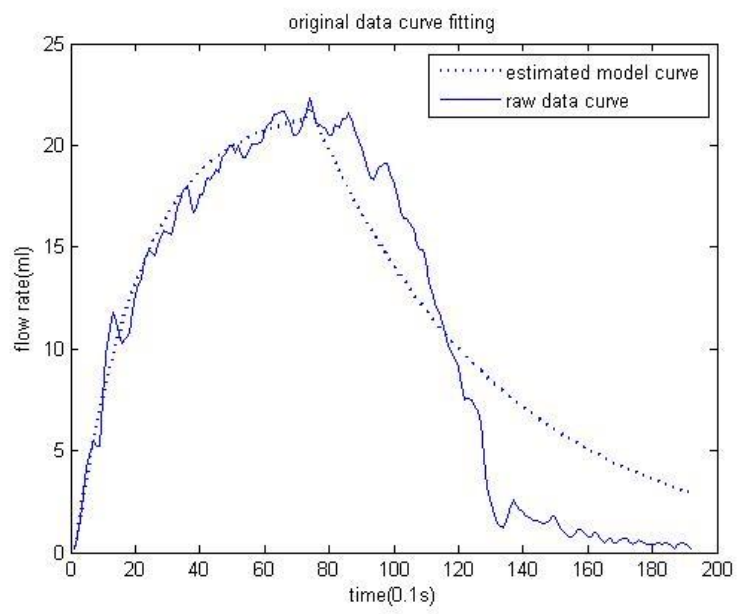

Figure 2 original curve and estimated model plot

\section{Filter design and data processing}

\subsection{Filter design}

In the real urodynamic tests, during micturition process there are fluctuations, caused by abdominal squeezing and bladder squeezing called artefacts, which may affect the 
their estimated characteristics, consequently produce incorrect diagnoses.

In the ICS (International Continence Society) guideline on urodynamic equipment performance [12], it states the clinical requirements for a standard urodynamic system to lead to technical recommendations, which also specified the frequency of abdominal squeezing and bladder squeezing. As a matter of fact, the specified frequencies provide guidance on selection of cut-off frequency in filter design to reduce noise effect.

There are several types of digital filters can be used to reduce signal noise. Compared to the other digital filters, Butterworth filter rolls off more slowly around the cut-off frequency than Chebyshev filter or the Elliptic filter, but without ripple, which can effectively reduce the artificial noise but uttermost keeps the original characteristic in raw data. The two filter's frequency responses are shown in Figure 3.

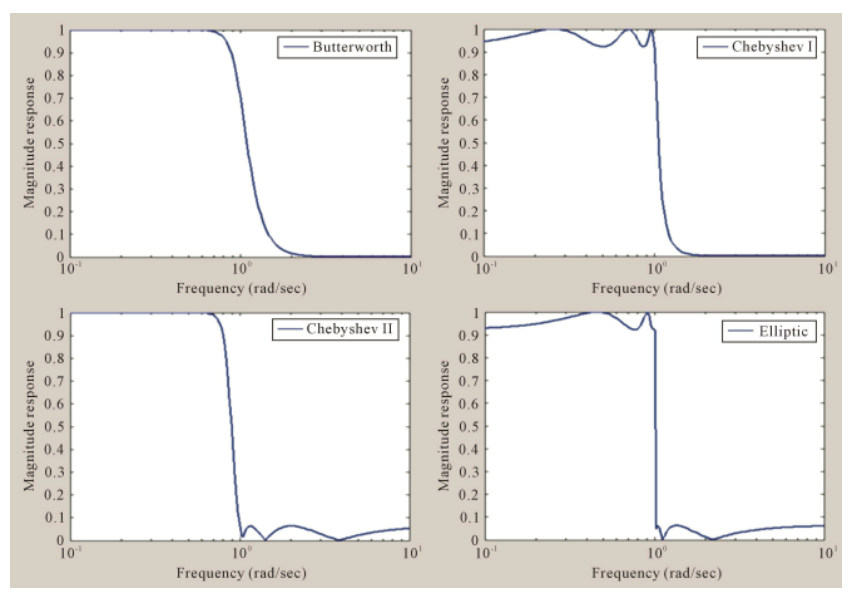

Figure 3 Butterworth comparison with other filters [13]

Therefore Butterworth filter can be considered as a suitable candidate for reducing the artefacts, which shows that a low pass filter could be designed whose cut-off frequency is normalized to 1 radian per second and whose frequency response (gain) is

$$
G(\omega)=\sqrt{\frac{1}{1+\omega^{2 n}}}
$$

Where $\omega$ is the angular frequency in radians per second and $\mathrm{n}$ is the number of poles in the filter-equal to the number of reactive elements in a passive filter.

When used in forms of digital filter, a $Z$ transfer function of an n-order Butterworth low pass filter can be expressed as [14]

$$
H(z)=\frac{B(z)}{A(z)}=\frac{b_{1}+b_{2} z^{-1}+\cdots+b_{n+1} z^{-1}}{a_{1}+a_{2} z^{-1}+\cdots+a_{n+1} z^{-n}}
$$

where $n$ is the order of the filter.

Furthermore, the order selection of the filter has been proposed and tested according to the proposed model structure. In the initial test, the third order Butterworth filter has been chosen as it is a wildly used to effectively reduce the noise level. However in the after filtered real data processing there was shown negative value for the time constant but positive in the raw data, which means the urine flow rate shows an infinite trend. Since the urodynamic model is represented as a first order discrete time dynamic model, if the data after the third order filter estimated is fitted with a first order system, the result may be inaccurate and distort. Therefore the third order Butterworth filter was changed to the first order one.

In Matlab code design, the cut-off frequency $\omega_{n}$ must be $0.0<\omega_{n}<1.0$, with 1.0 corresponding to half the sample rate. The sampling rate in the urodynamic test is $10 \mathrm{~Hz}$, so the coefficient in Matlab filter design has inputted as 0.2 for $1 \mathrm{~Hz}$ filter to reduce abdominal squeezing fluctuation and 0.02 for $0.1 \mathrm{~Hz}$ filter to reduce bladder squeezing fluctuation. The digital filter transfer functions for two sessions have been identified below.

$$
\begin{aligned}
& H(z)_{1 H z}=\frac{0.2452+0.2452 z^{-1}}{1-0.5095 z^{-1}} \\
& H(z)_{0.1 H z}=\frac{0.0305+0.0305 z^{-1}}{1-0.9391 z^{-1}}
\end{aligned}
$$

The frequency and phase response plots are shown below respectively.
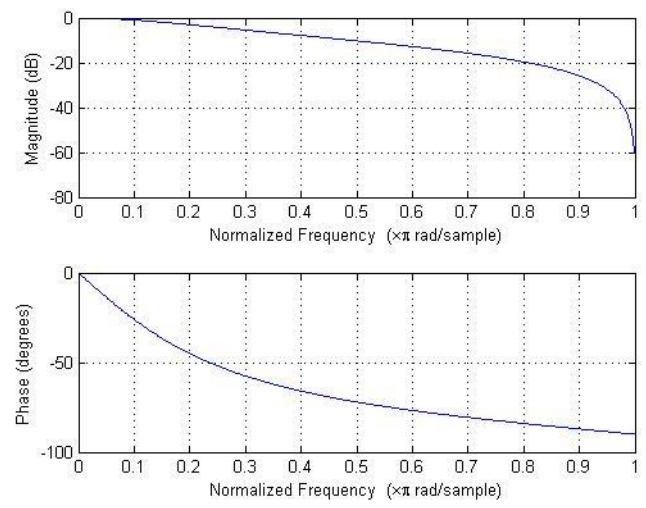

Figure 4 Frequency and phase response plots for $1 \mathrm{~Hz}$ filter
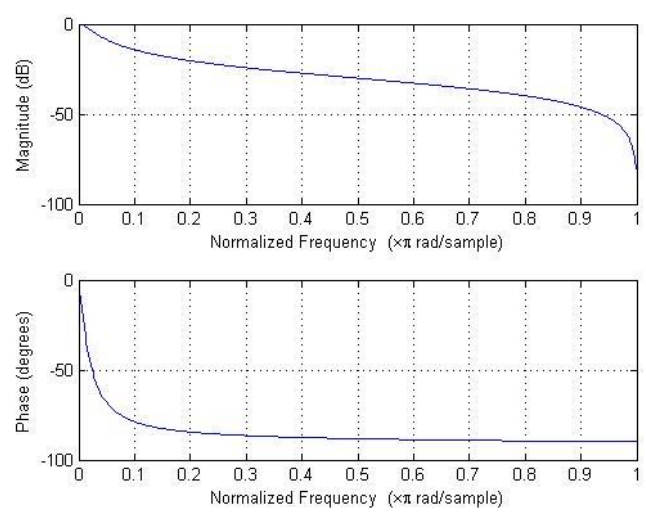

Figure 5 Frequency and phase response plots for $0.1 \mathrm{~Hz}$ filter

\subsection{Filter effectivity analysis}

Example 2 An original urine flow rate curve is shown in Figure 6. 


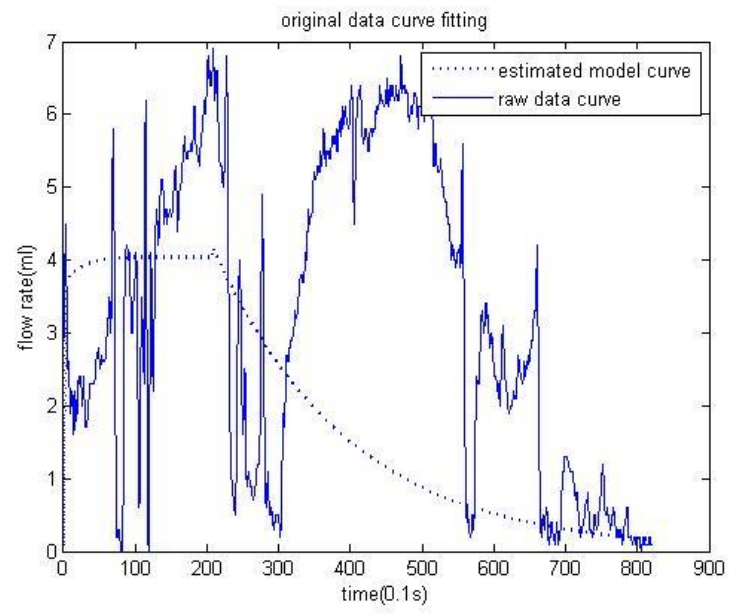

Figure 6 Original data and estimated model plot

This curve apparently has a large amount of fluctuation and interrupted urination in the half way of micturition process. The time constant values for the upward part and the downward part of estimated urine flow rate model are 23.6189 and 187.1409 respectively from the raw data sequence. It can be observed the max value has been chosen around the point 200, at the first half part of urination.

The filtered data and estimated urine flow rate model plot shows below.

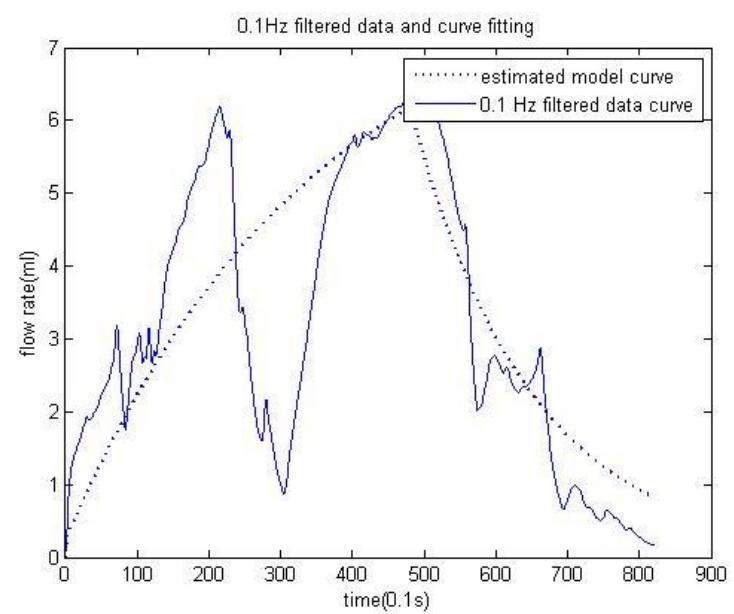

Figure $70.1 \mathrm{~Hz}$ filtered urine flow rate curve and estimated model plot

After $0.1 \mathrm{~Hz}$ filter, the max value point has been chosen by the time around 500 , just with small curve changing that the maximum value goes down from 6.9 to 6.8 . The value for time constant in the upward part and down ward part of the estimated filtered urine flow rate model are 343.5801 and 168.2998 respectively, showing a drastically changing from the original data's upward part time constant value. The reason for this can be recognised as the curve has been marginally changed and the ridge value has been re-chosen as well. If take an analysis with the raw data, since the interrupted urination happened, meanwhile the main urine flow came in the second half part. Therefore the max point should be chosen in the second half part instead of the point almost at the start of urination.

The data processing results show that the fluctuation can be effectively reduced and the proposed urine flow rate model and the filer design can be successfully implemented for the urine flow rate modelling tasks. Furthermore, it can be recognized using the model after filter is more reliable than the raw data estimated model.

\section{Analysis of peak account}

To have further analysis of the after filtered, it can be proposed another quantitative parameter for diagnosing disease if the number of peaks in the original data and after reducing fluctuations data can be compared. In this research, the peak point is defined as a data sample is larger than the neighbouring samples and greater than $1 \mathrm{ml}$ to avoiding tiny fluctuation induced by urodynamic measurement equipment.



Figure 8 Peak numbers per 100ml comparison between raw data and filtered data

Taking analyse of the peak account between raw data and filtered data from 10 patient's data, it can be observed from Figure 8 the blocks of normal patient locate in the left bottom corner of the figure, and the blocks of underactive and obstructed patients locate above or around the diagonal line and below the diagonal line respectively. It should be noted that this is currently happening under 10 samples, the further analysis of more patients' data need to be surveyed.

\section{Conclusions and future work}

This paper has proposed an empirical procedure for modelling and analysis of measured urine flow rate data. Although it is developed for medical engineering applications, the study has paid more attention to the aspects in data processing and modelling. Applications for urine flow rate research and diagnosis will be reported in the other follow up papers.

It should be clearly indicated that the new procedure is still under development and due to insufficient patients' measured data. There are a number of issues, associated with measurement of urine rate processes, to be accommodated in the future studies. It has been observed that during the real data processing there are several special cases, which show a large amount of fluctuation characteristic, need to be comprehensively studied. And the other characteristic parameters should be further studied, which cannot be obtained by the current procedure, but could be used for medical diagnosing or prediction. The future work will be focused on studies of more possible shape recognition/classification techniques, some of the combinational methodologies to cover global model and local models. Wavelet analysis will be considered as a tool. 


\section{References}

[1] Hosein, R.A. and Griffiths, D.J., (1990) Computer simulation of the neural control of bladder and urethra, Neurourol Urodyn, Vol. 9, No. 6, 601-618, 1990.

[2] Bastiaanssen. E.H., van Leeuwen, J.L., Vanderschoot, J. and Redert, P.A., A myocybernetic model of the lower urinary tract, J Theor Biol, Vol. 178, No. 6, 113-133. 1996.

[3] Hbener, U. and van Mastrigt, R., Computer simulation of micturition, Urodinamica, Vol. 4, No. 6, 81-90, 1994.

[4] van Duin, F., Rosier, P.F., Rijkhoff. N.J., van Kerrebroek, P.E., Debruyne, F.M. and Wijkstra, H., A computer model of the neural control of the lower urinary tract, Neurourol Urodyn, Vol. 17, No. 3, 175-196, 1998.

[5] Verdini, L., Biscotto, S., Costantini, E., Mearini, E. and Verdini, L., Perugia urodynamic method of analysis (PUMA): a new advanced method of urodynamic analysis applied clinically and compared with other advanced methods, Neurourol Urodyn, Vol. 22, No. 6, 206-222, 2003.

[6] Valentini, F.A., Besson, G.R., Nelson, P.P. and Zimmern, P.E., Clinically Relevant Modeling of Urodynamics Function: The VBN Model, Neurourology and Urodynamics, Vol. 33, No. 3, 51-57, 2013.

[7] Valentini, F.A., Besson, G.R., Nelson, P.P. and Zimmern, P.E., A mathematical micturition model to restore simple flow recordings in healthy and symptomatic individuals and enhance uroflow interpretation, Neurourol Urodyn, Vol. 19, No. 2, 153-176, 2000.

[8] Valentini, F.A., Zimmern, P.P., Besson, G.R. and Nelson, P.P., Modelized analysis of pressure flow studies of patients with lower urinary tract symptoms (LUTS) due to benign prostatic enlargement (BPE), Neurourol Urodyn, Vol. 22, No. 1, 45-53, 2003.

[9] Rollema, H. and Mastrigt, R., Objective analysis of prostatism: A clinical application of the computer program CLIM, Neurourology and Urodynamics, Vol. 10, No. 1, 7176, 1991

[10] Soderstrom, T. and Stoica, P., System identification (1st ed.), Prentice Hall International, Hemel Hempstead, UK, 1989.

[11] Ogata, K., Modern control engineering (4th ed.), Prentice Hall International, London, UK, 2002.

[12] Gammie, A., Clarkson, B., Constantinou, C., Damaser, M., Drinnan, M., Geleijnse, G., Griffiths, D., Rosier, P., Schäfer, W., van Mastrigt, R. and International Continence Society Urodynamic Equipment Working Group, International Continence Society guidelines on urodynamic equipment performance, Neurourology and Urodynamics, Vol. 33, No. 4, 370-379, 2014.

[13] Laghari, W.M., Baloch, M.U., Mengal, M.A. and Shah, S.J., Performance Analysis of Analog Butterworth Low Pass Filter as Compared to Chebyshev Type-I Filter, Chebyshev Type-II Filter and Elliptical Filter, Circuits and Systems, Vol. 5, 209-216, 2014.

[14] Williams, A.B., Electronic filter design handbook (4th ed.), McGraw-Hill, New York, London, 2006. 